\title{
The Application of a Polynomial Chaos Approach to the Vibration Analysis of an Elevator System
}

\author{
Diego Colón ${ }^{1}$, Stefan Kaczmarczyk ${ }^{2}$, and José M. Balthazar ${ }^{3}$ \\ ${ }^{1}$ Laboratório de Automação e Controle (LAC -PTC) - Escola Politécnica da Universidade de São Paulo, Av. Prof. Luciano \\ Gualberto, travessa 3, n 158, CEP 05508-900, São Paulo, BRAZIL, E-mail: diego@lac.usp.br \\ 2 The University of Northampton, School of Science and Technology, St George's Avenue, Northampton, NN2 6JD, \\ UNITED KINGDOM, E-mail: Stefan.Kaczmarczyk@northampton.ac.uk \\ 3 Instituto Tecnológico da Aeronáutica (ITA), Divisão de Mecânica e Aeronáutica, Praça Marechal Eduardo Gomes, 50 - \\ Vila das Acácias, CEP 12.228-900, São José dos Campos, BRAZIL, E-mail: jmbaltha@ita.br.
}

Abstract: The paper presents the uncertainty quantification of an elevator system by the Method of Polynomial Chaos. Initially, the mathematical model (the equations of motion) of an elevator system is derived and the sources of randomness are identified, which are in the elevator's trail. The method of polynomial chaos is then presented in its two versions (intrusive and non-intrusive) and the non-intrusive version is applied (numerically) in order to evaluate the uncertainties in the amplitude of the rope and car vibration by means of a auto-covariance function.

Keywords: Elevator Systems, Polynomial Chaos, Uncertainty Quantification

\section{INTRODUCTION}

Elevator systems have several important applications from office building to industrial transportation of material and goods. In Fig. 1, one can see a typical elevator system schematics, where the car (plus load) travels in a trail, that is normally a source of vibrations to the system as the car moves up/down (Kaczmarczyk and Iwankiewicz, 2006). A counterweight helps to counterbalance the car in any position along the trail. A traction sheave apply torque to the system, that is responsible for the car's motion. Brakes are used to balance the system when it stops in a floor (and for safety reasons). The traveling cables deliver electrical energy and communication to the car. The traction sheave is investigated in Arrasate et al. (2014), where several control techniques are approached. It is expected that a good velocity control of the traction sheave can be achieved, which guarantees that the mathematical model of the system can be decoupled and the car.

Polynomial Chaos is a technique to solve stochastic differential equations in which the system's solution (which is a stochastic process) is expanded in a base of orthogonal polynomials, that depends on the random parameters of the system. The polynomial chaos approach has two different methods to obtain the solution:

1. The intrusive method, in which it is obtained an associated deterministic system of differential equations for the time-varying coefficients of the expansion. This system has high dimension and must be solved numerically, in general;

2. The non-intrusive method, in which those same coefficients are obtained by using the definition of inner product in the polynomial space, that is related to the expected value operator. In this method, solving integrals is more critical than solving differential equations.

In Colón et al. (2014a,b), the intrusive method is applied to practical control problems, in which robustness analysis are conducted real plants and its controllers, designed with classical techniques. Other references also apply the method to control problems (Fisher and Bhattacharya, 2008; Smith et al., 2006; Xiu and Karniadakis, 2002). If $p$ is the number of polynomials in the orthogonal basis, and $n$ is the stochastic system's dimension, the associated deterministic system has order $n p$, which represents a very heavy load to the computational system. Although both methods (intrusive and non-intrusive) are equivalent theoretically, they are very different in the numerical solution. Results in the literature, and our own experience, reveals that the non-intrusive method is more efficient numerically. Also, the time necessary to construct the associated deterministic system is considerable, which is not necessary in the non-intrusive method (Xiong et al., 2014). Other works corroborate our conclusions, pointing out that the non-intrusive solution is more convenient (Sudret, 2008; Eldred, 2009).

In this work, we present the application of the polynomial chaos technique to an elevator system in which the car submitted to an stochastic horizontal disturbance, that comes to irregularities in the trail's profile. We follow the line presented in Kaczmarczyk and Iwankiewicz (2006). A mathematical model for the horizontal model of the system, some concepts of polynomial chaos are presented, as well as numerical results for the problem. 


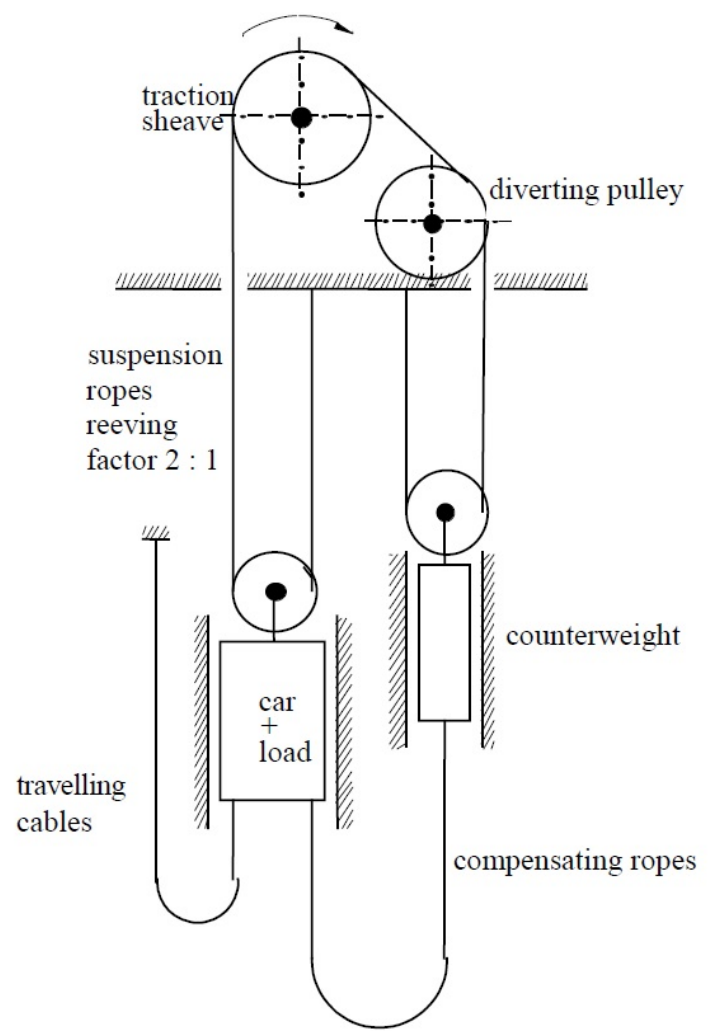

Figure 1 - Typical Elevator Installation.

\section{MATHEMATICAL MODEL}

In Kaczmarczyk and Iwankiewicz (2006), the authors claim that a properly designed control system for the longitudinal (up/down) motion can decouple the motion of the car and the counterweight. Figure 2 shows a simplified schematics of the decoupled elevator system in order to determine the mathematical equations that describes the lateral motion of the car + load caused by the nonuniform profile of the trail. It is easy to see that the suspension ropes have the same linear velocity as the car. The coupling between the trail and the car is represented by a spring of constant $k$. One point of the spring varies according to the profile $s(l)$ (that depends on a vertical coordinate $l$ ). The rope is under tension $T(x, t)$ and its horizontal motion is described by the function $w(x, t)$, where $x$ is another vertical coordinate. The two boundary conditions are $w(0, t)=0$ and $w(L, t)$, which is car's horizontal position. The rope's linear specific mass is $m$. The complete mathematical model for the horizontal vibration of the car can then be considered as a distributed model with a discrete mass in one end, but with the length slowly variable. The rope's length (from the traction sheave to the load) is represented by $L(t)$. If we define the parameter slow time factor $\epsilon=V / \omega_{0} L_{0}$, for the practical cases, we have that $\epsilon<<1$, and we can define the slow time $\tau=\epsilon t$ in order to clearly separate the dynamics related to the motion of the car (and variation of the rope's length) and its horizontal vibration. The time derivative of $L$ is related to the slow time derivative by $\dot{L}=\epsilon \mathrm{d} L / \mathrm{d} \tau=\epsilon L^{\prime}$. The complete system's dynamical equation assumes the form:

$$
m\left(w_{t t}+2 \epsilon L^{\prime} w_{x t}+\epsilon^{2}\left(L^{\prime}\right)^{2} w_{x x}+\epsilon^{2} L^{\prime \prime} w_{x}\right)+\gamma\left(w_{t}+\epsilon L^{\prime} w_{x}\right)-\left(T w_{x}\right)_{x}=0
$$

The boundary condition in the car end is given by:

$$
M\left(w_{t t}+2 \epsilon L^{\prime} w_{x t}+\epsilon^{2}\left(L^{\prime}\right)^{2} w_{x x}+\epsilon^{2} L^{\prime \prime} w_{x}\right)_{x=L}+k(w-s)_{x=L}+\left(T w_{x}\right)_{x=L}=0
$$

A more detailed analysis of the modeling process can be found in Kaczmarczyk and Iwankiewicz (2006). We search for solutions of the form:

$$
w(x, t)=\sum_{n=1}^{N} \Psi_{n}(x, \tau) q_{n}(t)
$$

where $\tau$ is slow time. We can search for functions of the form $\Psi_{n}(x, \tau)=\sin \left(\beta_{n}(\tau) x\right)$, where the wave-number $\beta_{n}(\tau)$, that is slowly variable in time, satisfy the equation: 


$$
\left(k-\frac{M}{m} T(\tau) \beta_{n}^{2}\right) \sin \left(\beta_{n} L(\tau)\right)+T(\tau) \beta_{n}\left(\cos \beta_{n} L(\tau)\right)=0
$$

where $T(\tau)=[M+(1 / 2) m L(\tau)] g$ is the time-varying mean tension.

After a substitution, we obtain a set of ordinary differential equation for the time-varying coefficients of the form:

$$
\ddot{q}_{r}(t)+2 \varsigma_{r} \omega_{r}(\tau) \dot{q}_{r}(t)+\omega_{r}^{2}(\tau) q_{r}(t)=\frac{\Psi_{r}(L(\tau))}{m_{r}(\tau)} k s(t)
$$

where $r=1,2 \cdots, N$ and

$$
m_{r}(\tau)=m \int_{0}^{L} \Psi_{r}^{2}(x, \tau) \mathrm{d} x+M \Psi_{r}^{2}(L)
$$

The wave-numbers $\beta_{n}$ are related to the natural frequencies of the several modes by $\omega_{n}(\tau)=\bar{c} \beta_{n}(\tau)$, where $\bar{c}=$ $\sqrt{T(\tau) / m}$ represents the time-dependent velocity of the lateral wave.

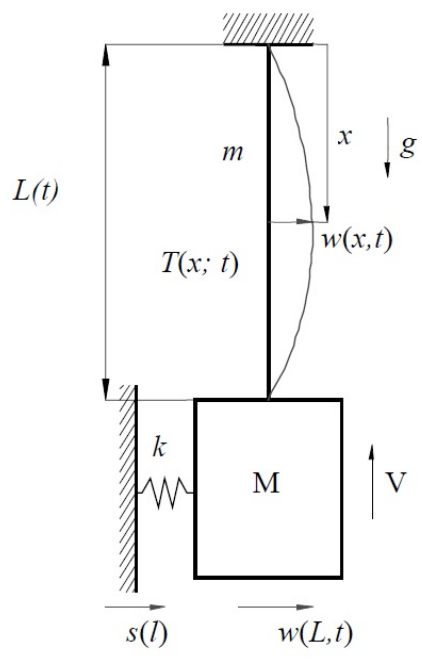

Figure 2 - Elevator System Mathematical Model.

The set of equations in Eq.4 are decoupled in the sense that the vibration modes are independent of one another. The equations are linear, but (slowly) time-varying with a damping coefficient $\varsigma_{r}$ for the $r$ natural mode. For a very slowly vertical motion, a frequency response analysis can be carried out by supposing that the trail's profile $s(l)$ can be represented by a Fourier series/transform.

\section{Stochastic equations}

It appears more reasonable to suppose that $s(l)$ has a random nature, as the trail fabrication can involve several random factors. Also, the natural degradation of the trail due to environmental factors (like oxidation) changes its format in nonpredictable ways. Finally, several sources of vibration (for example, small seismic disturbances and shape's variation coming from the effect of the wind in the building) are also present. In the present analysis, it is supposed that the trail shape $s(l)$ is a Gaussian stochastic process (colored noise) with zero-mean and autocorrelation function given by:

$$
K_{s}(\lambda)=\sigma_{s}^{2} e^{-\alpha|\lambda|}
$$

where $\sigma_{s}^{2}$ is the variance of $s$, and $\lambda=l_{2}-l_{1}$. This means that the stochastic process $s(l)$ satisfies the Itô equation given by $\mathrm{d} s=-\alpha s \mathrm{~d} l+\sigma_{s} \sqrt{2 \alpha} \mathrm{d} W(l)$, where $W(l)$ is the Wiener process. The complete stochastic system is given by Eq.5 below:

$$
\left\{\begin{aligned}
\mathrm{d} q_{r} & =\dot{q}_{r} \mathrm{~d} t \\
\mathrm{~d} \dot{q}_{r} & =-\omega_{r}^{2} q_{r} \mathrm{~d} t-2 \varsigma \omega_{r} \dot{q}_{r} \mathrm{~d} t+\frac{\Psi_{r}(L(\tau))}{m_{r}(\tau)} k s(t) \mathrm{d} t \\
\mathrm{~d} s & =-\alpha s V \mathrm{~d} t+\sigma_{s} \sqrt{2 \alpha V} \mathrm{~d} W(t)
\end{aligned}\right.
$$


where the scaling property of the Wiener process was used (Øksendal, 2003). Solutions for this systems are vectors of stochastic processes. We can see that the greater the parameter $\alpha$ the lesser the correlation between different points in the trail.

In Kaczmarczyk and Iwankiewicz (2006), it is analyzed an explicit solutions of Eq.5 by using Itô's stochastic calculus. This technique, on the other hand, is very difficult and non-intuitive to carry on, and can become very difficult to apply. The reader interested can find more on this procedure in $\varnothing \mathrm{ksendal}$ (2003). Numerical solutions, on the other hand, could be used, specially if we are interested in the statistics associated to the problem (probability distributions, mean values, variances and the like). Any numerical procedure that we can use to find such results must use an approximation of the Wiener process $W(t)$ as a combination of a finite number of random variables $\Delta_{1}, \cdots, \Delta_{N}$ (a good candidate is the Karhunen-Loeve (KL) approximation, that will be explained below). Monte Carlo (MC) simulation is a traditional way to obtain such results, which consists in sampling the parameter space and simulate the system for those values. The samples are obtained by a pseudo-random number generation. The several data obtained by the multiple simulation process are used for calculations that almost always involves integration (or numerical quadrature). If $N$ samples are selected, the rate of convergence is of order $\mathcal{O}(1 / \sqrt{N})$, and is independent of the dimension of the problem, that is, the number of independent random parameters in the system. Quasi-Monte Carlo techniques, that use low discrepancy sequences, has integration errors of order $\mathcal{O}\left(c N^{-\alpha}\right)$, where $\alpha=0.5$ is for standard MC, but this performance degrades as the problem dimension increases and the objective function's smoothness decreases (Morokoff and Caflisch, 1995).

\section{POLYNOMIAL CHAOS METHOD}

In this section, we present some theoretical aspects of the Polynomial Chaos Method. Given a measurable space as $(\Omega, \mathcal{F})$, where $\Omega$ is the sample space and $\mathcal{F}$ its $\sigma$-algebra (family of measurable sets), and $P$ a probability measure in this space, any measurable function $f: \Omega \rightarrow \mathbb{R}$ is a random variable (in the Borel $\sigma$-algebra of $\mathbb{R}$ ). A stochastic process is a function $g: T \times \Omega \rightarrow \mathbb{R}^{m}$, represented by $g(t, \omega)$, with $\omega \in \Omega$, in which, for each $t \in T, g_{t}(\omega)$ is a random variable, and $g_{\omega}(t)$ is a trajectory of the stochastic process. A stochastic process can also be viewed as a new sample space $\tilde{\Omega}$ where the points are the trajectories of the process, and the $\sigma$-algebra is formed by measurable sets of these trajectories, as can be seen in $\varnothing \mathrm{ksendal}$ (2003). An appropriate measure is this space completes the specification of the stochastic process.

The Hermite polynomials (in dimension one) satisfy the following relation (Spiegel, 1974):

$$
\int_{-\infty}^{\infty} e^{-\frac{x^{2}}{2}} H_{m}(x) H_{n}(x) \mathrm{d} x=\left\{\begin{array}{cc}
0 & m \neq n \\
n ! \sqrt{2 \pi} & m=n
\end{array}\right.
$$

If the inner product in the Hilbert space of functions $\mathcal{L}_{2}(\mathbb{R})$ is defined by the integral in Eq.6, where the weight function is $\rho(x)=e^{-\frac{x^{2}}{2}}$, then the polynomials $H_{n}(x)$ form an orthogonal basis in this space. Some examples of Hermite polynomials are $H_{0}(x)=1, H_{1}(x)=x, H_{2}(x)=x^{2}-1, H_{3}(x)=x^{3}-3 x$. There are also recursive formulas that can be used to calculate the set of normalized Hermite polynomials, that is $H_{n+1}(x)-x H_{n}(x)+n H_{n-1}(x)=0$.

The polynomial chaos theory proves that it is possible to express stochastic processes that depends on a finite number of random variables $\Delta_{1}(\omega), \Delta_{2}(\omega), \cdots, \Delta_{n}(\omega)$ (Cameron and Martin, 1947; Ghanem and Spanos, 1993), that is:

$$
x(t, \omega)=x\left(t ; \Delta_{1}, \Delta_{2}, \cdots, \Delta_{n}\right)=\Gamma_{0}(t)+\sum_{i=1}^{n} \Gamma_{i}\left(t, \Delta_{i}\right)+\sum_{i=1}^{n-1} \sum_{i<j}^{n} \Gamma_{i j}\left(t, \Delta_{i}, \Delta_{j}\right)+\cdots
$$

where $\Gamma_{0}(t)$ is the expected value of the stochastic process, and $\Gamma_{i}(t)=E\left[x(t) \mid \Delta_{i}\right]-\Gamma_{0}(t)$

In the simplest case, where all the random variables and stochastic process depends on (or is approximated by) only one random variable $\Delta$, we have:

$$
f(\Delta)=\sum_{n=0}^{\infty} f_{n} H_{n}(\Delta), \text { where } f_{n}=\int_{-\infty}^{\infty} f(\Delta) H_{n}(\Delta) \rho(\Delta) \mathrm{d} \Delta
$$

and it is easy to show that $E\left(N_{0}(\Delta)\right)=1$ and $E\left(N_{n}(\Delta)\right)=0$.

It would be severely restrictive a theory if it only worked for Gaussian processes. In fact, for other probability measures, similar polynomial expansions can be done, with the same numerical advantages of Hermite case. In those cases, different families of polynomials must be used, that relates probability density functions (PDF) and the corresponding orthogonal polynomials. Those families of polynomial functions are known as Wiener-Askey scheme, and are presented in ?. All this polynomials form basis in the space $\mathcal{L}_{2}(\Omega, \mathcal{F}, P)$, which is also the space of finite variance random variables. 


\section{Intrusive Polynomial Chaos}

In many applications, it is possible to find explicit solutions to the system of stochastic equations (Øksendal, 2003; Elliott, 1982; McKean et al., 2014). Essentially, as explained by Sussmann (1978), if every stochastic process in the equation could be written as a function $f: \Omega \times T \rightarrow \mathbb{R}^{m}$, the system could be solved, in the $t$ variable, for each $\omega \in \Omega$, with the same methods used for deterministic systems. On the other hand, the situation is far more complex when white noise is involved, as it is not a traditional stochastic process (the correct way to represent is by means of stochastic distributions, as explained in Øksendal (2003)). In this case, Itô calculus should be applied. In order to illustrate the application of the MPC to the analysis and solution of stochastic ordinary differential equations (which is necessary in robustness analysis), it is supposed that the system has one random parameter $\Delta(\omega)$ (Fagiano and Khammash, 2012), that is:

$$
\dot{x}_{t}=F\left(x_{t}, \Delta, t\right)
$$

where $t$ is the time variables. The general solution is of the form:

$$
x(t ; \omega)=\sum_{i=0}^{\infty} x_{i}(t) \phi_{i}(\omega)
$$

where $\left\{\phi_{i}(\omega)\right\}$ is a basis for the Hilbert space $\mathcal{L}_{2}(\Omega, \mathcal{F}, P)$, that are random variables in function of $\Delta$ (so are dependent variables). The functions/coefficients $\left\{x_{i}(t)\right\}$ are then deterministic functions. In particular, the solution of the system in Eq.8 must have such a decomposition, and $\phi_{i}(\omega)$ must be functions in $\mathcal{L}_{2}(\Omega, \mathcal{F}, P)$. If the solution is numerical, some statistical parameters are commonly calculated, as for example means, variance, standard deviations and estimates for PDF (probability density functions). In particular, the time-variant mean is given by $x_{0}(t)$ and the variance is given by:

$$
\operatorname{Var}[x(t ; \omega)]=E\left[x^{2}\right]-(E[x])^{2}=\sum_{i=0}^{\infty} x_{i}^{2} E\left[\phi_{i}^{2}(\Delta)\right]-x_{0}^{2}
$$

Normally, the procedure to find the resultant equations is too complex to be done manually, and some symbolic manipulation software should be used. In fact, a very complex software was coded by the authors in order to perform the analysis, which should not be rewritten for each new system that one wants to analyze. Nevertheless, for the sake of illustration, we show the 'manual' determination of the resultant system for the stochastic equation $\dot{x}=a x^{2}$, where $a$ is a random variable. The general solution is of the form:

$$
x_{i}(t, \Delta)=\sum_{m=0}^{p-1} x_{i, m}(t) \phi_{m}(\Delta)
$$

where $p$ is the number of polynomials in the Galerkin (truncated) basis. The time derivative of the states, that is $\dot{x}_{i}$, only affects the non-random part, that is $\dot{x}_{i}(t, \Delta)=\sum_{m=0}^{p-1} \dot{x}_{i, m}(t) \phi_{m}(\Delta)$. After some substitutions in the state equations, and using orthogonality properties, a set of deterministic ordinary differential equation are reached. The integration of such system produces some special realizations of the solution stochastic process. All the other realizations can be calculated from those. In fact, for the case at hand, we have:

$$
\sum_{m=0}^{p-1} \dot{x}_{m}(t) \phi_{m}(\Delta)=\sum_{m=0}^{p-1} a_{m} \phi_{m}(\Delta) \sum_{i=0}^{p-1} x_{i}(t) \phi_{i}(\Delta) \sum_{j=0}^{p-1} x_{j}(t) \phi_{j}(\Delta)
$$

After the projection in the direction of polynomial $\phi_{r}(\Delta)$, we have:

$$
\begin{gathered}
\dot{x}_{r}\left\langle\phi_{r}, \phi_{r}\right\rangle=\left\langle\sum_{m=0}^{p-1} \dot{x}_{m} \phi_{m}, \phi_{r}\right\rangle=\sum_{m=0}^{p-1} \sum_{i=0}^{p-1} \sum_{j=0}^{p-1} a_{m} x_{i} x_{j}\left\langle\phi_{m} \phi_{i} \phi_{j}, \phi_{r}\right\rangle \\
\dot{x}_{r}=\sum_{m=0}^{p-1} \sum_{i=0}^{p-1} \sum_{j=0}^{p-1} a_{m} x_{i} x_{j} \frac{\left\langle\phi_{m} \phi_{i} \phi_{j}, \phi_{r}\right\rangle}{\left\langle\phi_{r}, \phi_{r}\right\rangle}
\end{gathered}
$$

that is, each equations was transformed in $p$ equations, and each term in the sum was transformed in $p^{3}$ terms (in fact the number is smaller, as the multi-index $e_{m i j, r}=\frac{\left\langle\phi_{m} \phi_{i} \phi_{j}, \phi_{r}\right\rangle}{\left\langle\phi_{r}, \phi_{r}\right\rangle}$ possesses several symmetries). The set of deterministic equations must then be integrated (initial value problem). 


\section{Non-intrusive Polynomial Chaos}

Some observations about the intrusive method follows:

1. The order of the system of the expansion coefficients is $n p$ and it is nonlinear, and no special structure is known, which can difficult a lot the numerical solution;

2. The method can only be applied to polynomial systems, that is, the state variable appears in polynomial form in the stochastic differential equations, as well as the random parameters. Eventual transcendental functions must be approximated by finite sum of polynomials, as for example Taylor polynomials;

3. The number of terms in the expanded (deterministic) equations can grow very fast with the number of polynomials in the truncated expansion;

4. There is a considerable difficulty in finding the system in Eq.13 before the numerical integration even for polynomial systems, and sparsity of the multi-index coefficients should be explored.

A different approach for the problem consists in finding the expansion coefficients not by solving a system of deterministic differential equations in Eq.13, but by using the inner product formula:

$$
x_{i}\left(t_{k}\right)=\frac{E\left[x\left(t_{k}, \omega\right) \phi_{i}(\omega)\right]}{E\left[\phi_{i}(\omega) \phi_{i}(\omega)\right]}
$$

where the denominator is calculated by an explicit formula, the numerator is calculated by sampling $\Omega$, solving the original system for each sample (to obtain $x\left(t_{k}\right)$ ), and apply multidimensional quadrature methods to calculate the numerator. This must be done to each time value $t$ in which we are interested. This method is known as the non-intrusive method, as no previous modification of the stochastic system must be done in order to proceed to the coefficient calculations (Xiong et al., 2014). The original differential equations must be solved by some samples of the parameters, but the number of samples are much less than the used in the Monte Carlo method, and no modification of the system is necessary. Besides, those sample simulations can be done in parallel, which could be well explored in a parallel computation system, like clusters or GPU (graphic processing units) based systems.

Let the event space $\Omega=\mathbb{R}^{d}$, where $d$ is the number of independent random variables in the system. If in each dimension there are $m$ sample points, the total number of samples is $m^{d}$, which is again the curse of dimensionality. In order to avoid this problem, we could use:

1. LHS (Latin Hypercube Sampling): which consists in partitioning the sample space $\Omega$ in equiprobable sets and conveniently sampling in a way that each set is sampled, or;

2. Use the Sparse Grid Integration, which consists in selecting convenient grids to $\Omega$ in which the number of point does not grow exponentially with the dimension, like $m^{d}$, but in a much slower growing rate.

Both methods provides efficient ways to calculate the numerator in Eq.14, but there are differences in the convergence rate between them. The LHS method has the advantage of to have been deduced using probabilistic concepts. The sparse grid method, on the other hand, appears to achieve a better rate of convergence, as the objective function's smoothness is increased. There are lots of papers in which comparative analysis are done, with theoretical limits presented, as well as numerical experiments. The reader interested in the details of the sparse grid approach can consult Gerstner and Griebel (1998). The non-intrusive polynomial chaos approach has other advantages over the intrusive, as presented in Xiong et al. (2014). In this reference, the author present a comparative analysis for some simple systems with only one source of randomness (one single random parameter).

\section{NUMERICAL RESULTS}

In order to find numerical solutions to the system, one must find an approximation to the stochastic process $s(l)$ by means of a finite number of random variables. A possible solution would be a truncated Karhunen-Loeve series of the form:

$$
s_{N}(l)=\sum_{i=1}^{N} c_{i} \phi_{i}(l)
$$

where $\phi_{i}(l)$ are functions of $l$ that form a basis to the space $L^{2}(0, L)$ of square-integrable (or finite energy) signals, and $c_{i}$ are random Fourier coefficients that are uncorrelated, that is $E\left[c_{i} c_{j}\right]=0$ for $i \neq j$. We do a Karhunen-Loeve expansion for the Wiener process $W(t)$ in Eq.5, which will produce the system: 


$$
\left\{\begin{array}{l}
\dot{x}_{r}=y_{r} \\
\dot{y}_{r}=-\omega_{r}^{2} x_{r}-2 \varsigma \omega_{r} y_{r}+\frac{\Psi_{r}(L(\tau))}{m_{r}(\tau)} k s(t) \\
\dot{s}=-\alpha s V+\sigma_{s} \sqrt{2 \alpha V} F\left(\Delta_{1}, \cdots, \Delta_{N}\right)
\end{array}\right.
$$

where we used $x_{r}=q_{r}$ and $y_{r}=\dot{q}_{r}$ to avoid confusion. Also, as the measure $\mathrm{d} W$ was approximated by a KL combination of random variables, the equations can be written as traditional differential equations, where $F\left(\Delta_{1}, \cdots, \Delta_{2}\right)$ is an approximation to the White Noise (Øksendal, 2003). The reader could question if this finite approximation in Eq.15 for the system in Eq.5 is the correct one, as according to Sussmann (1978), only Stratonovich stochastic differential equations has this property. In fact, according to Øksendal (2003), the Itô and Stratonovich integrals are equivalent in some situations, and that is the case in Eq.5.

We will consider the effects of the excitation to the fundamental mode (that is, $r=1$ ), and in this case, the damping ratio is will be considered $\varsigma_{1}=0.4$. The stiffness coefficient for the car-rail interface is $k=2083 \mathrm{~N} / \mathrm{m}$, and $\sigma_{s}=$ 7.07.10 $0^{-4} \mathrm{~mm}$. Obviously, the fundamental frequency and wave-number are slowly-varying, as shown before. We suppose that that rope's specific mass is $m=0.65 \mathrm{~m} / \mathrm{kg}$, the total car's weight (car + load) is $M=3250 \mathrm{~kg}$, the well height of the instalation is $70 \mathrm{~m}$, the car height is $3.2 \mathrm{~m}$ and the total travel height is $60 \mathrm{~m}$, which is also the maximum length of the rope $L_{0}$. We suppose two different vertical/longitudinal constant velocities $V$ for the car/rope, that are 1.5 $\mathrm{m} / \mathrm{s}$ and $6.0 \mathrm{~m} / \mathrm{s}$. It is also supposed that the elevator is ascending in the two simulations. This means that the rope has the slowly variable length $L(\tau)=(V / \epsilon) \tau+L_{0}$, and $\epsilon=\left(V / \omega_{0} L_{0}\right)$ is the slow time constant factor. In order to find the corresponding natural frequency, Eq.3 must be solved for each $\tau$.

Figure 3 shows important parameters for the problem when the elevator is ascending with vertical velocity $V=1.5$ $\mathrm{m} / \mathrm{s}$. The simulations were conducted from the bottom (when the rope's length is $60 \mathrm{~m}$ till zero, which corresponds to the final (not slow) time $t_{f}=39.9$ seconds. We can see that the velocity of the wave propagation does not change too much, but the wake number $\beta_{1}$ approach infinity, as well as the fundamental frequency, when $L(\tau)$ approaches zero. The amplitude of the forcing term in Eq.4, that is $\frac{\Psi_{r}(L(\tau))}{m_{r}(\tau)} k$, also goes to infinity, which means that the forcing term has growing influence as the elevator approaches the uppermost part of the instalation.
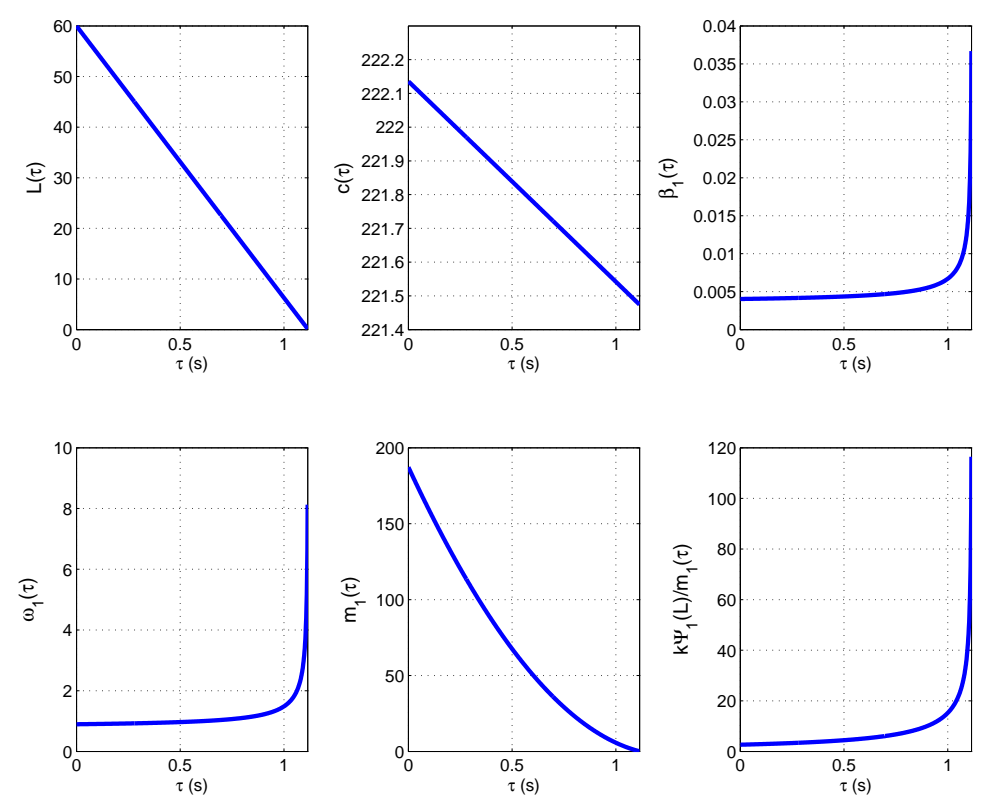

Figure 3 - Time-varying parameters for velocity $V=1.5 \mathrm{~m} / \mathrm{s}$

As can be found in Grigoriu (2013), a white noise $N(x)$, that has zero mean and correlation function $E(N(x) N(y))=$ $\gamma \delta(x-y)$, where $\delta(t)$ is the Dirac delta function, its KL representation is:

$$
N(x)=\sqrt{\gamma} \sum_{k=1}^{\infty} N_{k} \phi_{k}(x)
$$

where the functions $\phi_{k}(x)$ form an orthogonal set of functions, and $N_{k}$ has zero mean and $E\left(N_{k} N_{l}\right)=\delta_{k l}$, where $\delta_{k l}$ is 
the Kronecker delta and $\gamma$ is the intensity of the noise. The orthogonal functions in the white noise expansion $N(l)$ are $\left\{e^{j \frac{2 k \pi l}{L_{0}}}\right\}$, where $l \in \mathbb{Z}$ and $L_{0}$ is the period of the function. The Karhunen-Loeve expansion is then (with unit variance):

$$
N(l)=\sum_{k=-\infty}^{+\infty} N_{k} e^{j \frac{2 k \pi l}{L_{0}}}
$$

where $\left\{N_{k}\right\}$ are a series complex random variables. For $N(l)$ real, it is necessary that $N_{k}^{*}=N_{-k}$. If we consider $N_{k}=a_{k}+j b_{k}$, we have that $N_{-k}=a_{k}-j b_{k}$, so it is possible to adopt that $k \in \mathbb{N}$. We also consider that $N_{0}$ is null in order to have zero mean in $N(l)$. We must adopt that the double series $\left\{a_{k}\right\}$ and $\left\{b_{k}\right\}$ has zero mean and $E\left[a_{l} a_{k}\right]=\delta_{l k}$, $E\left[b_{l} b_{k}\right]=\delta_{l k}$ and $E\left[a_{l} b_{k}\right]=0$.

If we suppose initially that only two parameters, that are $a_{1}, b_{1}$, which are Gaussian zero mean with unitary covariance matrix, the auto-covariance function of state $q_{1}$ is given by (if the expansions are truncated in degree $p$ for the polynomials and $\mu_{t_{n}}=E\left(q_{1}\left(t_{n}\right)\right)$, and $\left.\mu_{t_{m}}=E\left(q_{1}\left(t_{m}\right)\right)\right)$ :

$$
\begin{array}{r}
E\left(q_{1}\left(t_{n}\right) q_{1}\left(t_{m}\right)\right)-\mu_{t_{n}} \mu_{t_{m}}=E\left(\sum_{i+j \leq p} c_{i, j}\left(t_{n}\right) \phi_{i, j}\left(a_{1}, b_{1}\right) \sum_{k+l \leq p} c_{k, l}\left(t_{m}\right) \phi_{k, l}\left(a_{1}, b_{1}\right)\right)-\mu_{t_{n}} \mu_{t_{m}} \\
=\sum_{i+j \leq p} \sum_{k+l \leq p} c_{i, j}\left(t_{n}\right) c_{k, l}\left(t_{m}\right) E\left(\phi_{i, j}\left(a_{1}, b_{1}\right) \phi_{k, l}\left(a_{1}, b_{1}\right)\right)-\mu_{t_{n}} \mu_{t_{m}}= \\
=\sum_{i+j \leq p} c_{i, j}\left(t_{n}\right) c_{i, j}\left(t_{m}\right) E\left(H_{i}^{2}\left(a_{1}\right) H_{j}^{2}\left(a_{2}\right)\right)-\mu_{t_{n}} \mu_{t_{m}}=\sum_{i+j \leq p} c_{i, j}\left(t_{n}\right) c_{i, j}\left(t_{m}\right) 2 \pi i ! j !-\mu_{t_{n}} \mu_{t_{m}}
\end{array}
$$

An approximation for the autocovariance function for ascending car with $V=1.5 \mathrm{~m} / \mathrm{s}$ is presented in Fig. 4 . We can see that the less the rope's length $L$, the greater the covariance.

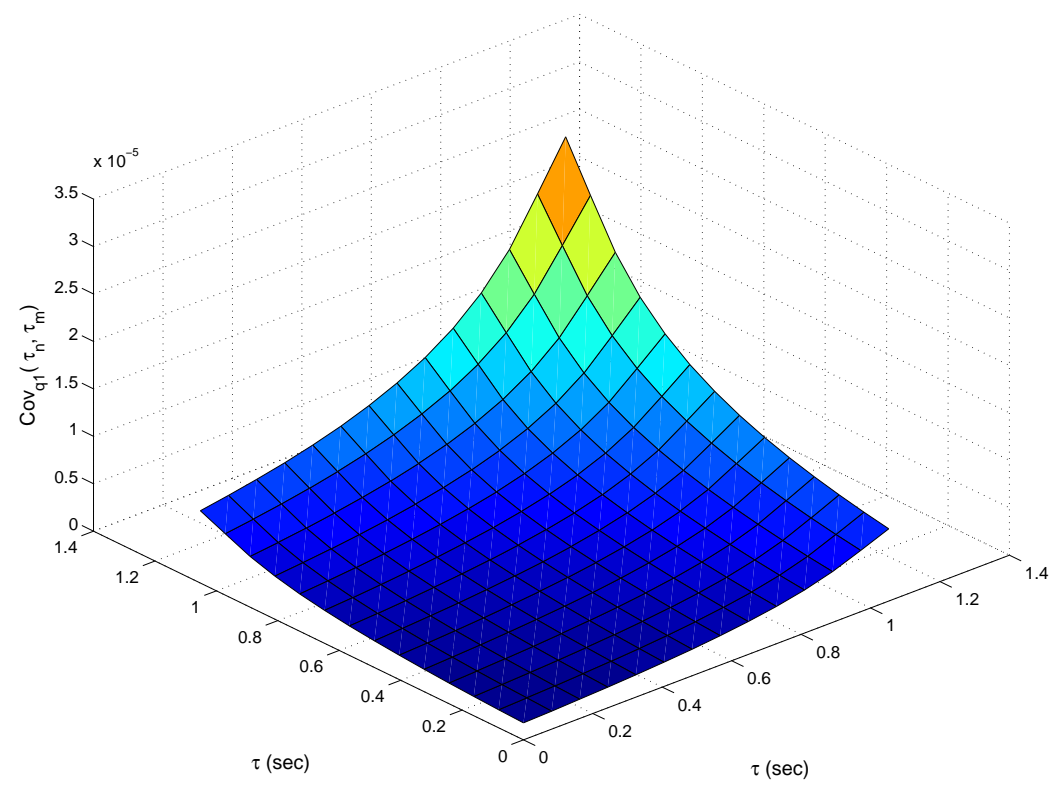

Figure 4 - Autocovariance Function for $V=1.5 \mathrm{~m} / \mathrm{s}$

For the case o ascending car with velocity $V 6.0 \mathrm{~m} / \mathrm{s}$, the calculations of $\epsilon$ and other parameters, and the resulting time-varying coefficients are shown in Fig. 5, and the autocovariance function is given in Fig. 6. We can see that the autocovariance is higher when $V=6.0 \mathrm{~m} / \mathrm{s}$ than when $V=1.5 \mathrm{~m} / \mathrm{s}$, which is consequence of the fact that the higher the velocity, the higher is the amplification of the noise that comes from the trail.

\section{CONCLUSION AND FUTURE WORK}

We presented an application of the polynomial chaos methodology for an elevator system in which the random rugosity of the trail causes vibration in the car's position. We could see that the higher velocities, the higher is the vibration. In fact, 

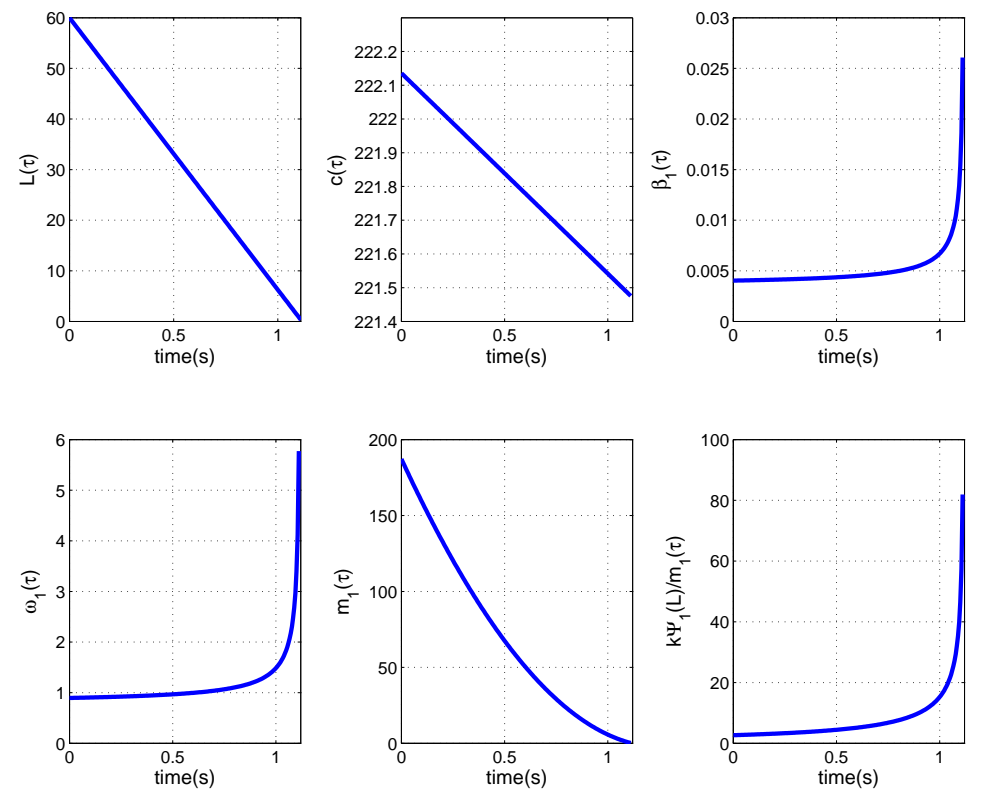

Figure 5 - Time-varying parameters for velocity $V=6.0 \mathrm{~m} / \mathbf{s}$

for the maximum variance, which occurs for $V=6 \mathrm{~m} / \mathrm{s}$ and almost zero length of the trail, the standard deviation, that is the square root of the variance, is about $9.5 \mathrm{~mm}$. The software developed in MATLAB took about five minutes to execute in a core I7 notebook with 4 GB of memory, as the the non-intrusive method was used (with sparse grid quadrature).

In future work, we intend to adopt a higher number of random parameters in the model (in the white noise approximation and other parameters of the system) and calculate other statistical data, as other moments and joint probability distributions. Also, a closed-loop control system to attenuate the effect of the disturbances will be proposed, that can compensate the vibrations in all the velocities and positions of the car, providing the same level of comfort in all situations.

\section{REFERENCES}

Xabier Arrasate, Stefan Kaczmarczyk, Gaizka Almandoz, José M. Abete, and Inge Isasa. The modelling, simulation and experimental testing of the dynamic responses of an elevator system. Mechanical Systems and Signal Processing, 42 (1-2):258 - 282, 2014. ISSN 0888-3270.

R. H. Cameron and W. Martin. The orthogonal development of non-linear functionals in series of fourier-hrmite functionals. Annals of Mathematics, Second Series, 48(2):385-392, April 1947.

Diego Colón, José M. Balthazar, Célia A. dos Reis, Átila M. Bueno, Ivando S. Diniz, and Suélia de S. R. F. Rosa. Control design and robustness analysis of a ball and plate system by using polynomial chaos. AIP Conference Proceedings, 1637:226-234, 2014a.

Diego Colón, Murillo A. S. Ferreira, José M. Balthazar, Átila M. Bueno, and Suélia de S. R. F. Rosa. Robustness analysis of an air heating plant and control law by using polynomial chaos. AIP Conference Proceedings, 1637:235-244, 2014b.

Michael Eldred. Recent advances in non-intrusive polynomial chaos and stochastic collocation methods for uncertainty analysis and design. In Structures, Structural Dynamics, and Materials and Co-located Conferences, pages -. American Institute of Aeronautics and Astronautics, May 2009.

R.J. Elliott. Stochastic Calculus and Applications. Applications of mathematics. Springer, 1982. ISBN 9780387907635.

L. Fagiano and M. Khammash. Simulation of stochastic systems via polynomial chaos expansions and convex optimization. ArXiv: Stat.CO, 1202.0753v3, november 2012.

James Fisher and Raktim Bhattacharya. Stability analysis of stochastic systems using polynomial chaos. In Proceedings of the 2008 American Control Conference, pages 4250-4255, 2008.

Thomas Gerstner and Michael Griebel. Numerical integration using sparse grids. Numerical Algorithms, 18(3-4):209232, 1998. ISSN 1017-1398. 


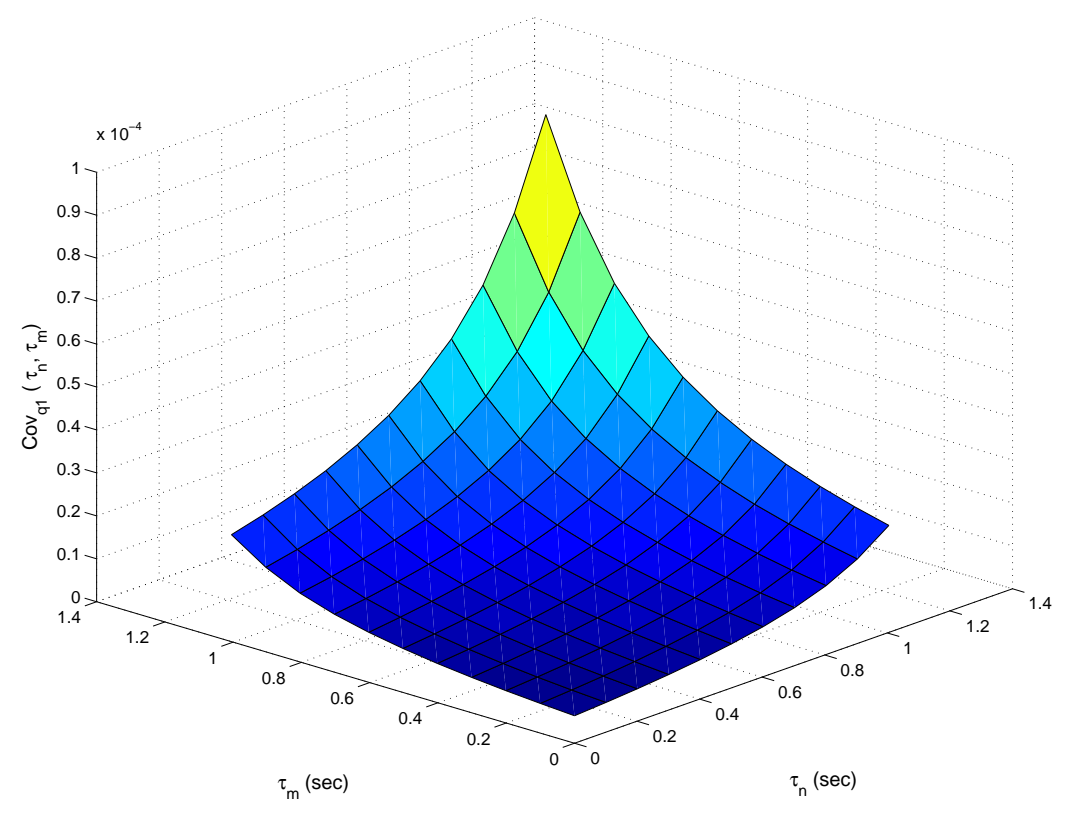

Figure 6 - Autocovariance Function for $V=6.0 \mathrm{~m} / \mathbf{s}$

R. Ghanem and P.D. Spanos. A stochastic galerkin expansion for nonlinear random vibration analysis. Probabilistic Engineering Mechanics, 8(34):255 - 264, 1993. ISSN 0266-8920.

M. Grigoriu. Stochastic Calculus: Applications in Science and Engineering. Birkhäuser Boston, $2013 . \quad$ ISBN 9781461265016.

Stefan Kaczmarczyk and Radoslaw Iwankiewicz. Dynamic response of an elevator car due to stochastic rail excitation. In Proceedings of the Estonian Academy of Sciences, volume 55, 2006.

H.P. McKean, Z.W. Birnbaum, and E. Lukacs. Stochastic Integrals. Probability and mathematical statistics. Elsevier Science, 2014. ISBN 9781483259239.

William J. Morokoff and Russel E. Caflisch. Quasi-monte carlo integration. Journal of Computational Physics, 122(2): 218 - 230, 1995. ISSN 0021-9991.

B. Øksendal. Stochastic Differential Equations: An Introduction with Applications. Hochschultext / Universitext. Springer, 2003. ISBN 9783540047582.

A. Smith, A. Monti, and F. Ponci. Robust controller using polynomial chaos theory. In Industry Applications Conference, 2006. 41st IAS Annual Meeting. Conference Record of the 2006 IEEE, volume 5, pages 2511-2517, Oct 2006.

Murray Spiegel. Fourier Analysis: with applications to boundary value problems. Schaum's Outline. McGraw-Hill, march 1974.

Bruno Sudret. Global sensitivity analysis using polynomial chaos expansions. Reliability Engineering \& System Safety, 93(7):964 - 979, 2008. ISSN 0951-8320. Bayesian Networks in Dependability.

Hector J. Sussmann. On the gap between deterministic and stochastic ordinary differential equations. The Annals of Probability, 6(1):19-41, 021978.

Fenfen Xiong, Shishi Chen, and Ying Xiong. Dynamic system uncertainty propagation using polynomial chaos. Chinese Journal of Aeronautics, 27(5):1156 - 1170, 2014. ISSN 1000-9361.

D. Xiu and G. E. Karniadakis. The wiener-askey polynomial chaos for stochastic differential equations. SIAM Journal of Scientific Computing, 24(2):619-644, 2002.

\section{RESPONSIBILITY NOTICE}

The author(s) is (are) the only responsible for the printed material included in this paper. 